\title{
Crosstalk Analysis under the Impact of Line-width and Chirp in 8×10 GBPS WDM System Incorporating Optical Cross-Connector
}

\author{
Vishal sharma \\ Shaheed Bhagat Singh State Technical Campus \\ Moga Road, Ferozepur
}

\author{
Jagvir Kaur \\ Shaheed Bhagat Singh State Technical Campus \\ Moga Road, Ferozepur
}

\begin{abstract}
In this paper, a simulative experimental set up for OWDM communication system consist of two 4- optical channels with channel spacing of $0.1 \mathrm{THz}$ interconnected by means of a $2 \times 2$ OXC (an OXC with 2 input ports and 2 output ports) to route the data with less delay and high throughput is analyzed under the impact of laser line-width and modulator's chirp over the crosstalk introduced in OWDM system. Our results show that by using $\mathrm{OXC}$, we can minimize the power degradation introduced in OWDM system due to crosstalk. In addition, the impact of laser line-width and modulator's chirp can not be ignored in reducing the crosstalk while dealing with long haul optical communication systems. The OWDM system is also reported under the influence of crosstalk at different bit rate varying from $1 \mathrm{Gbps}$ to $10 \mathrm{Gbps}$ in this work.
\end{abstract}

\section{General Terms}

Crosstalk Analysis

\section{Keywords}

Optical Cross-Connector (OXC), Crosstalk, Laser line-width, Modulator's chirp, Optsim simulation software

\section{INTRODUCTION}

Optical cross connects (OXCs) is an optical switch that can interconnect optical signals between multiple inputs and multiple outputs [1] and providing high switching speeds along with long- term reliability. Generally, electrical cross connects are attributed to the conversion of optical signals coming from the input optical fibers to the electronic signals in order to process the data, and followed by transformation of the electronic signals back to optical form to deliver them to the desired output fibers [2]. These conversions of the electronic to optical and optical to electronic may induce delay and crosstalk, especially in case of WDM/DWDM long haul optical systems. In contrast, OXCs are able to handle the optical signals in their native form and directly switch them from multiple input fibers to their desired output without the need of any optical to electronic conversion. Consequently, OXC greatly improves the switching throughput by reducing the crosstalk and delay. The OXCs are expected to play the important roles in the backbone of the fiber optical communication systems while electronic remains on the edges of the networks [3]. In OWDM systems, the crosstalk between wavelengths is a major problem that degrades the performance of the systems. It is found that the fiber nonlinearities lead to crosstalk between sub-carriers of different wavelengths traversing simultaneously through the fiber. Signal distortions in intensity-modulated direct detection WDM systems induced by interaction of fiber nonlinearity and dispersion were investigated [4]. Crosstalk between wavelengths in SCM-WDM optical communication systems has been studied [5]. It has been reported that in a dispersive fiber, crosstalk can be attributed to fiber nonlinearity combined with group velocity dispersion (GVD). Study of XPM- and SRS- induced crosstalk noise evolution as well as their interaction through a long lossy, non linear dispersive fiber in a cascaded IM-DD system was performed and it was found that it comes mainly from adjacent channels [6]. Power impairments and power penalty due to SRS in dispersion-managed fiber links were evaluated [7]. Transmission limitations due to XPM-induced crosstalk in SCM-DWDM systems were studied at wavelength spacing of 50- and 100- GHz [8]. The crosstalk of three optical cross connects based on Mach-Zehnder Interferometer (MZI) obtained at 4*10 Gbps WDM transmission through SSMF with $2 \times 2$ optical cross connects (OXC) is presented [9] but the impact of chirp and laser line-width of such systems are not reported. Further, this work is limited to only $40 \mathrm{Gbps}$ WDM systems only. An experimental setup is investigated to observe the amount of intensity noise introduced in an optical system and the power penalty required to compensate this intensity noise at different optical distances under the impact of spectral width of optical source of $10 \mathrm{Gbps}$ optical communication system under the individual and the combine impact of higherorder dispersion parameters [10]. This work is also limited to $10 \mathrm{Gbps}$ optical system and crosstalk analysis at high bit rate is not reported. In this paper, we demonstrated a simulative experimental set up consist of eight optical channels transmitted through SSMF by means of a $2 \times 2$ OXC to observe the impact of laser line-width and modulator's chirp over the power degradation introduced in an $8 * 10$ Gbps WDM system due to the crosstalk. The paper is organized into four sections. Section I presents the introduction followed by the section II that describe briefly about the simulation set-up of 8-channels OWDM system incorporating $2 \times 2$ OXC. The section III deals with the discussion of the results for the system based on optical cross connects at different modulator chirp parameter, laser line-width and bit rate. The section IV presents the conclusion drawn from our simulation results.

\section{SIMULATION SETUP}

The simulation set up schematically shown in Figure 1 consist of 8-channel WDM signals at different bit rate i.e. 1-/10-Gbps are modulated by means of external modulation technique using single arm Mach-Zehnder modulator over a continuous wave (CW) lasers with channel spacing of $0.1 \mathrm{THz}$ at varying laser line-width from $10 \mathrm{MHz}$ to $100 \mathrm{KHz}$ with $\mathrm{CW}$ power of 1 $\mathrm{mW}$. 


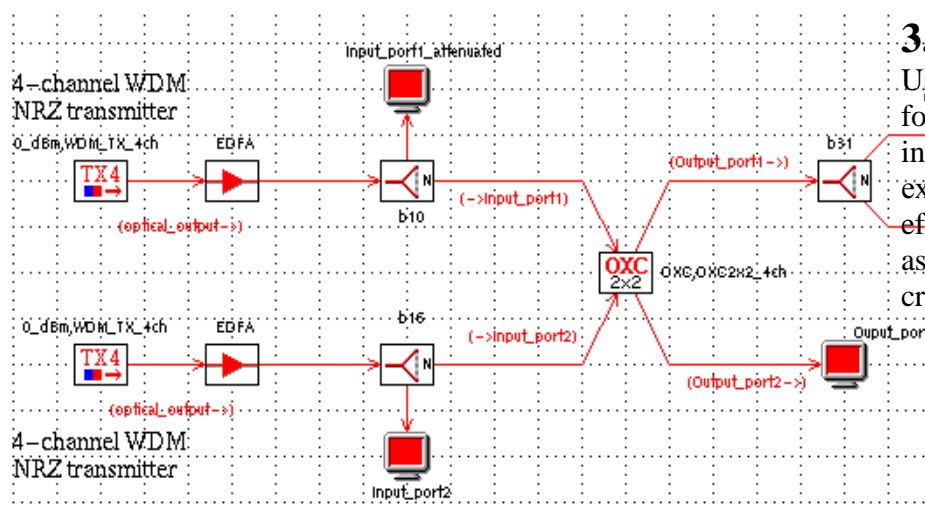

(a)

\section{RESULT AND DISCUSSION}

Using qur simulation set-up, the results have been mentioned for power degradations introduced due to crosstalk by incorporating OXC at different bit rate under the impact of exterinal modulator's chirp parameter and laser line-width. An effort has been made for the exhaustive investigation to ascefrin inthe impact of chirp parameter and laser line-width on crosstalk in WDM communication systems.
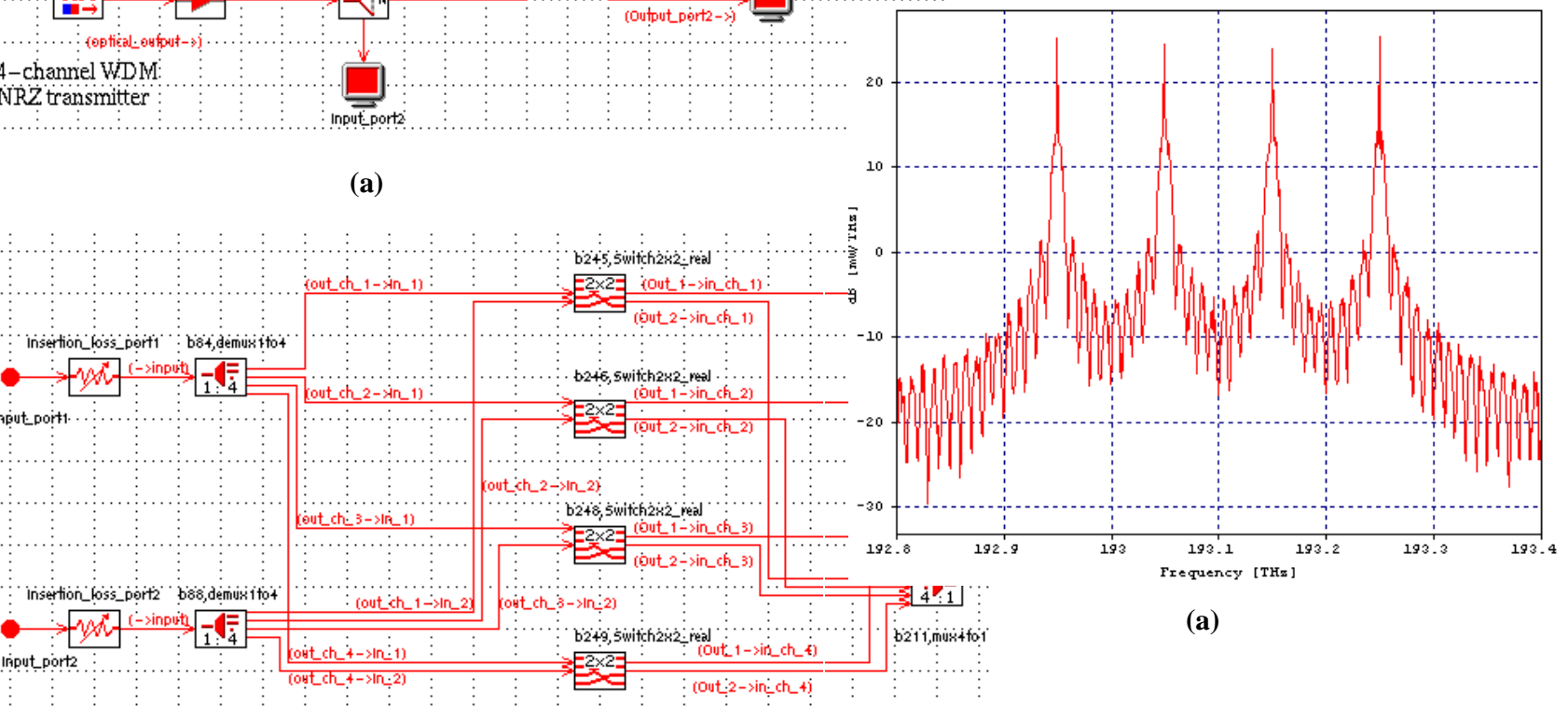

(b)

Fig 1: (a) Simulation setup of two 4- channel OWDM system using $2 \times 2$ OXC (b) Different sections of $2 \times 2$ OXC

\section{Table 1: System Parameters}

Center Frequency: 193.1THz

Channel Spacing: $0.1 \mathrm{THz}$

Filter Bandwidth: $5 \mathrm{GHz}$ Filter Roll Off: 0.2

Laser Input Power: $1 \mathrm{~mW}$

Insertion Loss: $2 \mathrm{~dB}$

Insertion loss switch: $0.5 \mathrm{~dB}$

Modulator Excess Loss: $3 \mathrm{~dB}$

Modulator Extinction Ratio: $30 \mathrm{~dB}$

These two set of four channels after amplification by using EDFA are interconnected by means of a $2 \times 2$ OXC that consist of three sections as de-multiplexer section that separates in frequency the optical channels of the WDM transmitted signals; realistic optical switches section that directs each channel towards an output port and multiplexer section that aggregates the channels at the output ports.

The optical power spectrum at each output port is analyzed by using Optical spectrum analyzer and optical power meter to observe and measure the cross-talk effect. The different system parameters used to simulate this system are tabulated in Table 1.

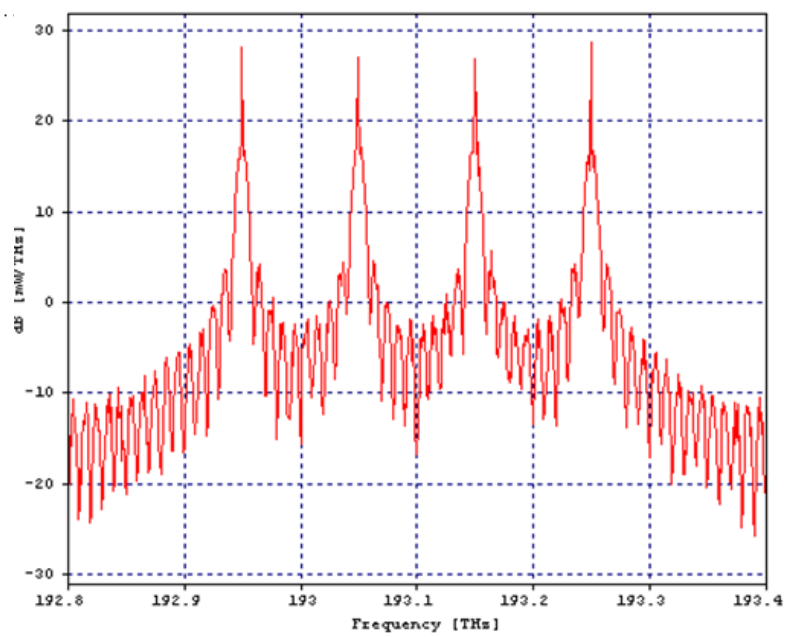

(b)

Fig: 2 Optical Spectrum before OXC of (a) input port 1 and (b) input port 2 with laser line-width $10 \mathrm{MHz}$ and zero chirp at 10Gbps

The Fig 2 depicts the input optical spectrum at the transmission end of port 1 and port 2 before interconnected to an OXC with laser line-width of $10 \mathrm{MHz}$ and modulator's chirp parameter is set to zero value. The 4-optical channels of each port with channel spacing of $0.1 \mathrm{THz}$ are transmitted at bit rate of 10 Gbps. The interference in the optical spectrum can be clearly seen from Fig 2. But as the crosstalk decreases from $0 \mathrm{~dB}$ to $50 \mathrm{~dB}$, this interference reduces as shown in Fig 3 after using OXC. Further, this crosstalk is also reduced if the channels are transmitted at bit rate of 1 Gbps as shown in Figs (4-5). Further, it is observed that the power degradation due to crosstalk introduced in the system increases as the crosstalk increases but 
reduces as laser line-width decreases as depicted in Fig 6. The Fig 7 depicts that not only the laser line-width and bit rate, the chirp parameter is also a dominant factor in reducing the crosstalk. As the chirp reduces from 3 to 0 , the power degradation due to crosstalk reduces as shown in Fig 7.

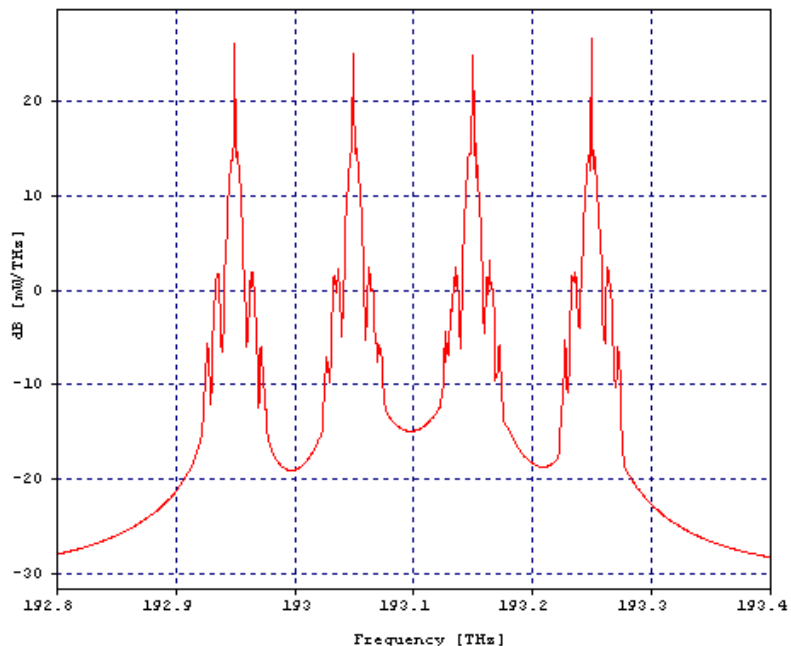

(a)

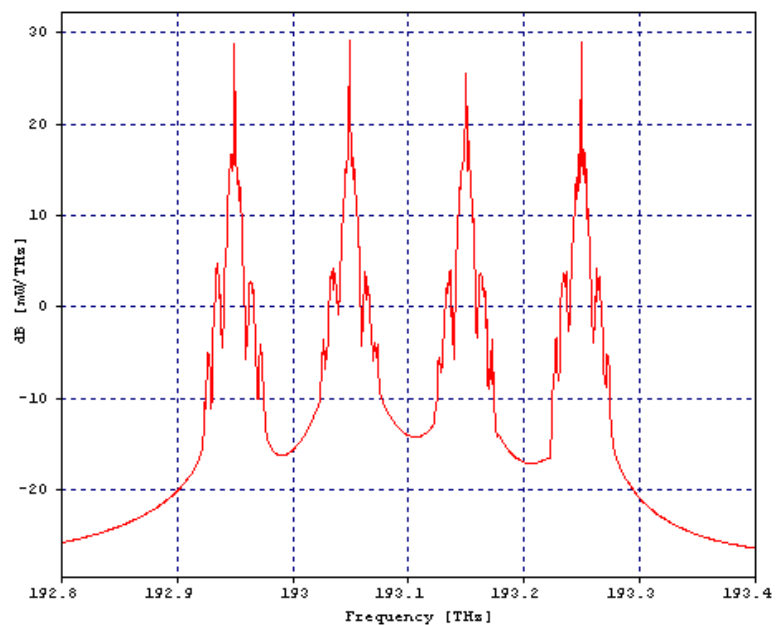

(b)

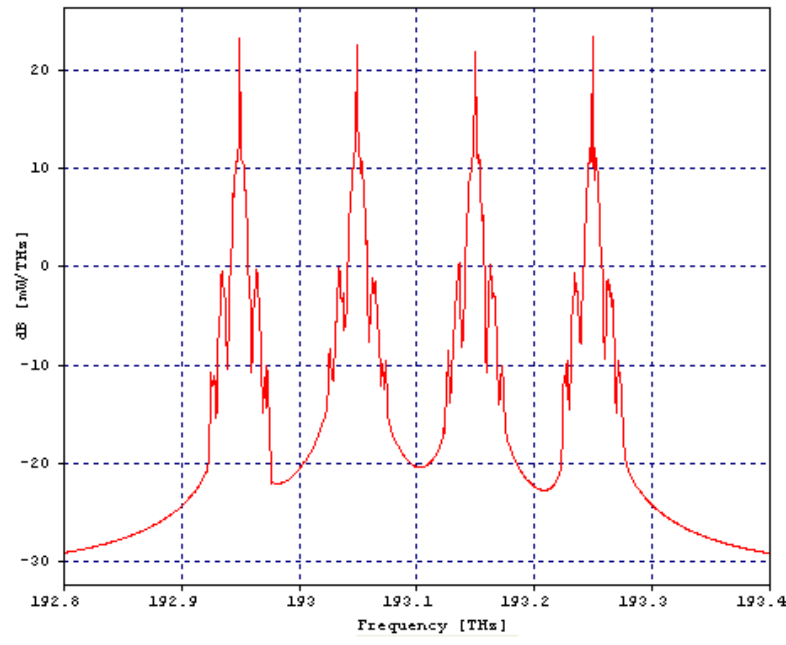

(c)

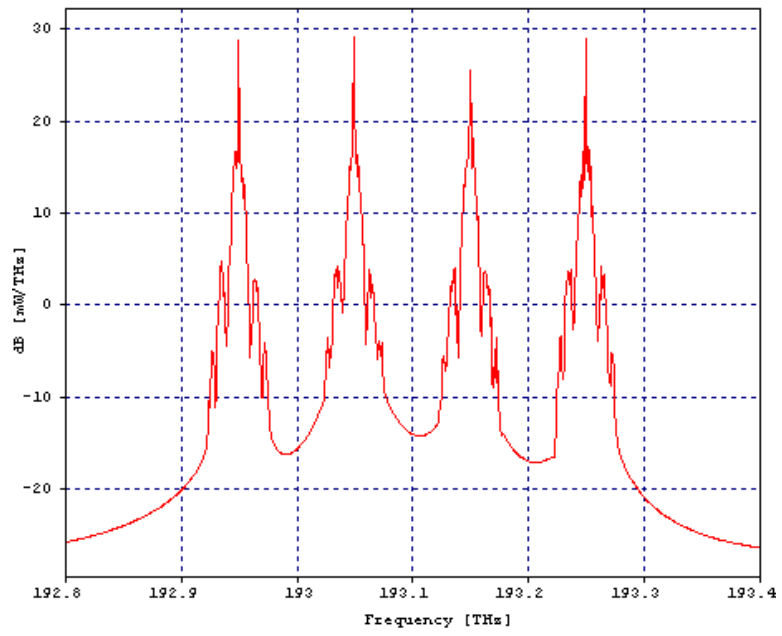

(d)

Fig: 3 Optical Spectrum after OXC of input port 1 with (a b) crosstalk $=-50 \mathrm{~dB}$ and of input port 2 with (c, d) crosstalk $=0 \mathrm{~dB}$ with laser line-width $10 \mathrm{MHz}$ and zero chirp at 10Gbps

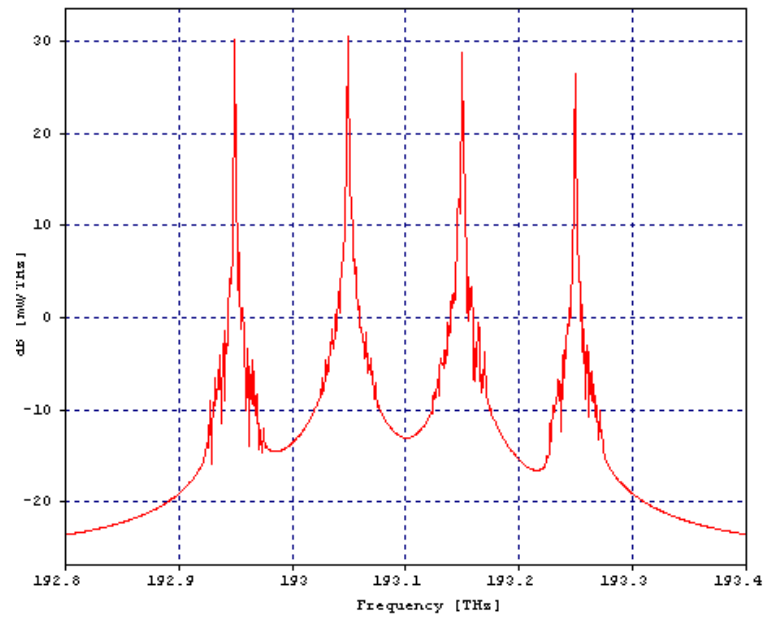

(a)

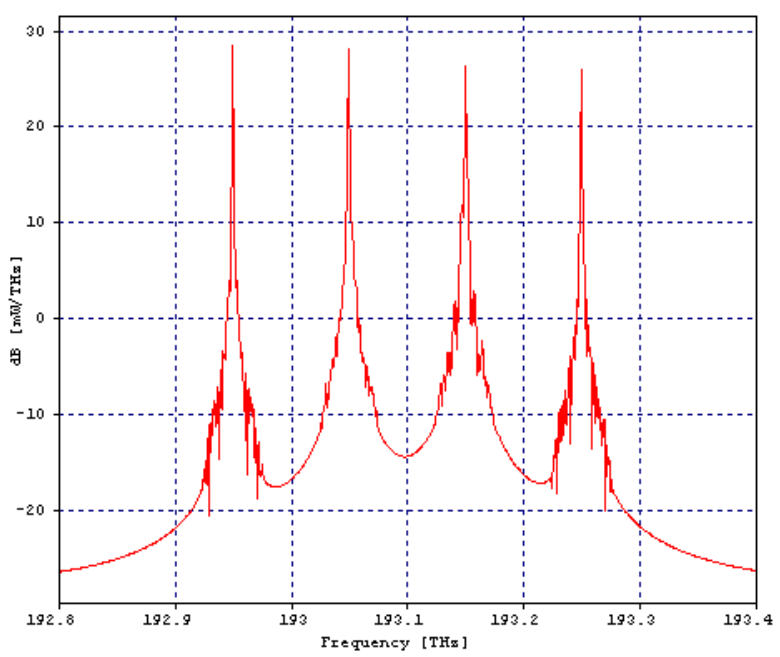

(b)

Fig: 4 Optical Spectrum after OXC of (a) input port 1 and (b) input port 2 with laser line-width $100 \mathrm{KHz}$, a zero chirp at $1 \mathrm{Gbps}$ at crosstalk $=0 \mathrm{~dB}$ 


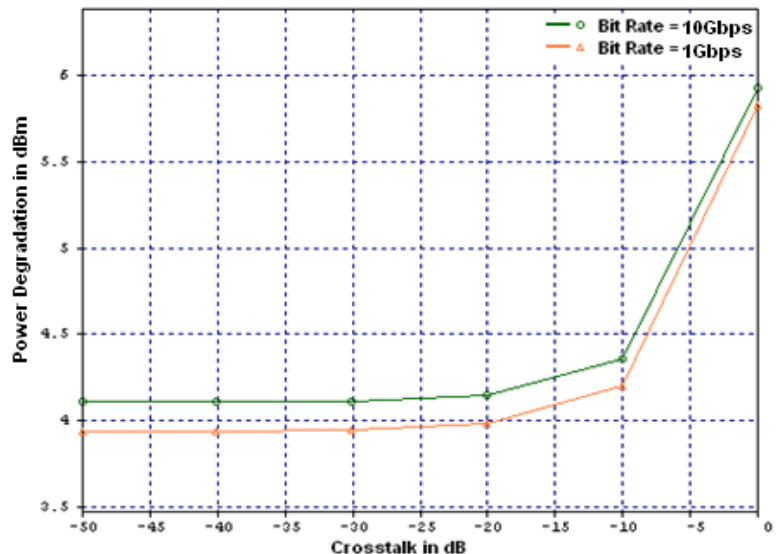

Fig: 5 Power degradation vs crosstalk at different bit rate with laser line-width of $100 \mathrm{KHz}$ at zero chirp

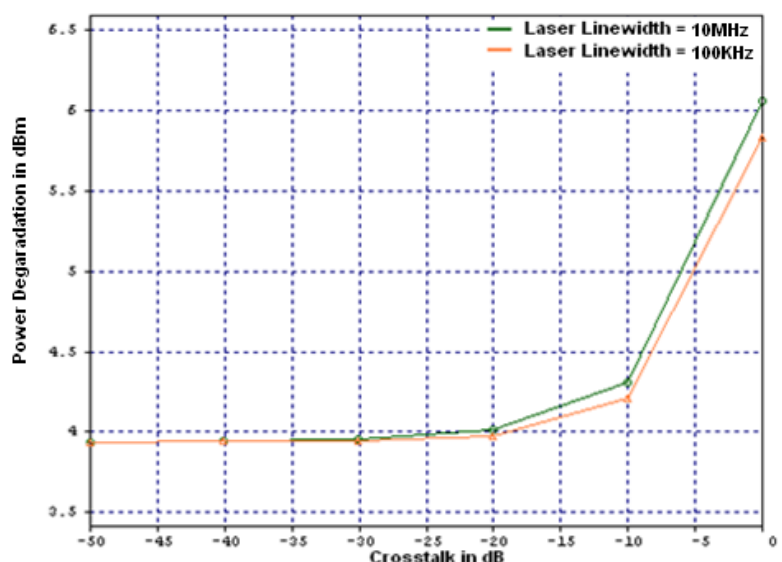

Fig: 6 Power degradation vs crosstalk at different laser line-width with zero chirp at bit rate of $10 \mathrm{Gbps}$

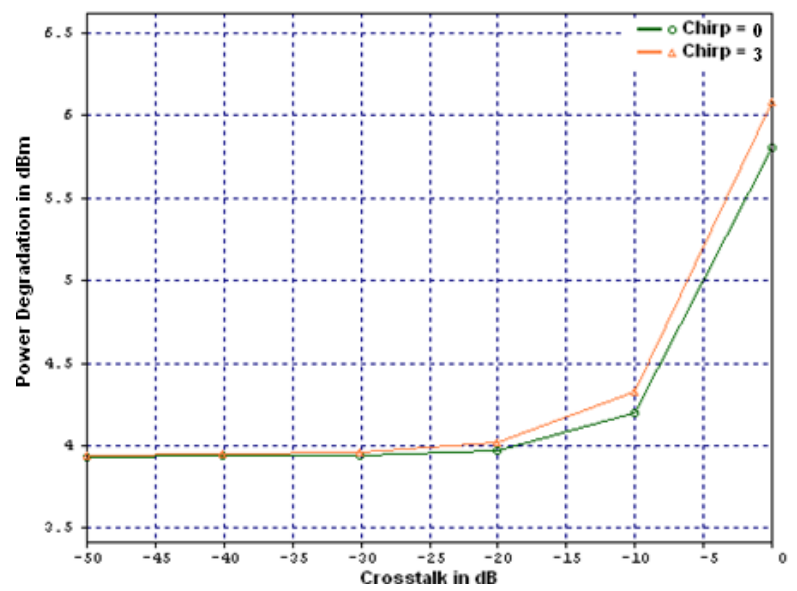

Fig: 7 Power degradation vs crosstalk at different chirp with laser line-width of $100 \mathrm{KHz}$ at bit rate of $10 \mathrm{Gbps}$

\section{CONCLUSION}

Demonstration of the impact of laser line-width and modulator's chirp over the crosstalk introduced in $8 * 10$ Gbps WDM system incorporating a $2 \times 2 \mathrm{OXC}$ is carried out in this paper, and observed a reduction of power degradation introduced in an 8-channel WDM system due to crosstalk. The crosstalk reduces as we reducing the laser line-width to 100 $\mathrm{KHz}$ in conjunction with the reduction of modulator's chirp.

\section{REFERENCES}

[1] S. Araki, S. Ganguly, R. Izmailov, Y. Maeno, Y. Suemura, T. Wang, "DWDM network, Hybrid cross-connects and hierarchical optical networks", NEC Res. Dev. 43 (2) (2002) 133-140.

[2] J. Hecht, "All optical networks need optical switches", Laser Focus World 5 (2000) 189-196.

[3] S. Clavenna, "Optical switching evolves beyond device market”, WDM Solutions 4 (2000) 10-12.

[4] Lutz Rapp, "Experimental investigation of signal distortions induced by cross- phase modulation combined with dispersion", IEEE Photon. Technol. Lett. 9 (12) (1997) 1592-1594

[5] Frank S.Yang, Michel E. Marhic, Leonid G. Kazovsky, "Non linear crosstalk and two counter measures in SCMWDM Optical communication systems", Journal Of Light wave Technology 18 (4) (2000) 512-520.

[6] Zhi Jiang, Chong Cheng Fan, "A comprehensive study on XPM- and SRS-induced noise in cascaded IM-DD optical fiber transmission systems", J. Light wave Technol. 21 (4) (2003) 953-960.

[7] Toshiaki Yamamoto, Seiji Norimatsu, "Statistical analysis on stimulated Raman crosstalk in dispersion-managed fiber links", J. Light wave Technol. 21 (10) (2003) 2229 2239.

[8] W. H. Chen, Winston I. Way, "Multichannel single-sideband SCM/DWDM transmission systems", J. Light wave Technol. 22 (7) (2004) 1679-1693.

[9] Sanjeev Dewraa, R.S. Kaler , "Crosstalk analysis in an optical network based on optical cross connects with different MZI techniques", Optik - International Journal for Light and Electron Optics, In Press, Corrected Proof, Available online 15 February 2012

[10] Vishal Sharma, Amarpal Singh, Ajay K Sharma, "Analysis and simulation of the effect of spectral width over intensity noise under the impact of higher-order dispersion parameters in the optical communication systems", Optics Communications, Volume 281, Issue 13, Pages 3495-3500, 2008 